\title{
The Role and Activation Mechanism of TAZ in Hierarchical Microgroove/Nanopore Topography- Mediated Regulation of Stem Cell Differentiation
}

This article was published in the following Dove Press journal: International Journal of Nanomedicine

\author{
Penghui Hu${ }^{1,2, *}$ \\ Qian Gao ${ }^{1,2, *}$ \\ Huimin Zheng ${ }^{1,2}$ \\ Yujuan Tian ${ }^{1,2}$ \\ Guoying Zheng' \\ Xiaoyu Yao' \\ Junjiang Zhang' \\ Xudong $\mathrm{Wu} \mathbb{( D}^{2}$ \\ Lei Sui (iD)
}

'Department of Prosthodontics, Tianjin Medical University School and Hospital of Stomatology, Tianjin, People's Republic of China; ${ }^{2}$ Department of Cell Biology, 201 I

Collaborative Innovation Center of

Tianjin for Medical Epigenetics, Tianjin

Key Laboratory of Medical Epigenetics,

Tianjin Medical University, Tianjin,

People's Republic of China

*These authors contributed equally to this work
Correspondence: Lei Sui

Department of Prosthodontics, Tianjin

Medical University School and Hospital of

Stomatology, Tianjin, People's Republic of

China

Email suilei@tmu.edu.cn
Purpose: To investigate the role and activation mechanism of TAZ in periodontal ligament stem cells (PDLSCs) perceiving hierarchical microgroove/nanopore topography.

Materials and Methods: Titanium surface with hierarchical microgroove/nanopore topography fabricated by selective laser melting combined with alkali heat treatment (SLMAHT) was used as experimental group, smooth titanium surface (Ti) and sandblasted, largegrit, acid-etched (SLA) titanium surface were employed as control groups. Alkaline phosphatase (ALP) activity assays, qRT-PCR, Western blotting, and immunofluorescence were carried out to evaluate the effect of SLM-AHT surface on PDLSC differentiation. Moreover, TAZ activation was investigated from the perspective of nuclear localization to transcriptional activity. TAZ knockdown PDLSCs were seeded on three titanium surfaces to detect osteogenesis- and adipogenesis-related gene expression levels. Immunofluorescence and Western blotting were employed to investigate the effect of the SLM-AHT surface on actin cytoskeletal polymerization and MAPK signaling pathway. Cytochalasin D and MAPK signaling pathway inhibitors were used to determine whether actin cytoskeletal polymerization and the MAPK signaling pathway were indispensable for TAZ activation.

Results: Our results showed that SLM-AHT surface had a greater potential to promote PDLSC osteogenic differentiation while inhibiting adipogenic differentiation than the other two groups. The nuclear localization and transcriptional activity of TAZ were strongly enhanced on the SLM-AHT surface. Moreover, after TAZ knockdown, the enhanced osteogenesis and decreased adipogenesis in SLM-AHT group could not be observed. In addition, SLM-AHT surface could promote actin cytoskeletal polymerization and upregulate p-ERK and p-p38 protein levels. After treatment with cytochalasin D and MAPK signaling pathway inhibitors, differences in the TAZ subcellular localization and transcriptional activity were no longer observed among the different titanium surfaces.

Conclusion: Our results demonstrated that actin cytoskeletal polymerization and MAPK signaling pathway activation triggered by SLM-AHT surface were essential for TAZ activation, which played a dominant role in SLM-AHT surface-induced stem cell fate decision.

Keywords: TAZ, hierarchical micro/nanoscale topography, periodontal ligament stem cells, osteogenic differentiation, adipogenic differentiation

\section{Introduction}

Mechanical signals that direct cell fate decisions include mechanical forces as well as the rigidity and surface topography of the extracellular matrix (ECM). ${ }^{1,2}$ Increasing evidence has shown that the surface topography of the matrix alone could effectively regulate stem cell differentiation. ${ }^{3-5}$ For intraosseous implants, the ideal surface 
topography should induce osteogenic differentiation of stem cells and consequently promote bonding of the implant to the surrounding bone tissue (osseointegration). Titanium surfaces with hierarchical micro/nanoscale topography were reported to enhance osteogenic differentiation while inhibiting adipogenic differentiation ${ }^{6-9}$ and are considered a promising alternative to the currently used microscale topography resulting from sandblasted, large-grit, and acidetched (SLA) surface treatment. Previously, we prepared a titanium surface with hierarchical microprotrusion/nanonotch topography using the direct metal laser sintering (DMLS) technique combined with acid etching and confirmed that it could improve osteogenesis. ${ }^{10}$ Then, we upgraded the preparation protocol and fabricated titanium surfaces with microgrooves/nanoporous hierarchical topography by selective laser melting (SLM) combined with alkali heat treatment (AHT), and our preliminary study suggested that this topography might be more effective in promoting the osteogenic differentiation of preosteoblast MC3T3-E1 cells than that of other surfaces. ${ }^{11}$ However, the underlying mechanism of hierarchical micro/nanoscale topography-mediated direction of stem cell differentiation remains unclear.

Yes-associated protein (YAP) and its paralog transcriptional coactivator with PDZ-binding motif (TAZ) are known as important mechanosensitive transcriptional regulators. ${ }^{12}$ Their activities and roles in signal transduction are closely related to their intracellular localization. ${ }^{13}$ In the cytoplasm, YAP/TAZ are found in an inactive phosphorylated state and are degraded by proteases. ${ }^{14}$ In the cell nuclei, YAP/TAZ converts to an active dephosphorylated state that is involved in various biological processes, such as cell cycle regulation, ${ }^{15}$ tumorigenesis, ${ }^{16}$ and transcriptional regulation. ${ }^{17}$ The $\mathrm{WW}$ domain in the TAZ structure was shown to bind to the Pro-Pro-X-Tyr sequences of the transcription factors RUNX2 and PPAR $\gamma$ to regulate cell differentiation. ${ }^{17,18}$ Recent studies have revealed that the intracellular localization of YAP/TAZ could be influenced by the material surface topography and was possibly involved in topography-mediated cell fate decisions. ${ }^{19-21}$ Jun-Ha Hwang reported that nanotube topography activated the mitogen-activated protein kinase (MAPK) pathway and thereby increased TAZ nuclear translocation. ${ }^{19}$ Houhua Pan demonstrated that the hierarchical macropore/nanowire topography increased YAP nuclear translocation to promote osteogenic differentiation. ${ }^{20}$ Accordingly, we hypothesized that YAP/TAZ also plays a critical role in the hierarchical microgroove/nanopore topography-mediated direction of stem cell fate.

Although several reports have shown that TAZ could be activated by titanium surfaces with hierarchical micro/ nanoscale topography, the activation mechanism of TAZ has yet to be studied. Current evidence on this issue suggests that the actin cytoskeleton and MAPK signaling pathway should be strongly considered. On the one hand, actin polymerization has been proven to be essential for TAZ nuclear localization. ${ }^{12}$ Previous reports indicated that hierarchical micro/nanoscale topography could promote actin cytoskeletal polymerization. ${ }^{10,22}$ Therefore, polymerization of the actin cytoskeleton may play an important role in the activation of TAZ. On the other hand, the MAPK signaling pathway has also been shown to be actively involved in mechanical cue-induced cell differentiation, ${ }^{23,24}$ including ECM stiffness-mediated differentiation through extracellular signal-regulated kinase (ERK) and c-Jun N-terminal kinase (JNK) activation ${ }^{25,26}$ and mechanical stress-induced differentiation via ERK1/2 and p38 activation. ${ }^{27,28}$ Moreover, the MAPK pathway was reported to be mechanically activated and subsequently influence the intracellular localization of YAP to regulate cell proliferation. ${ }^{29}$ These results indicated that the MAPK signaling pathway may also play a role in TAZ activation. Thus, we hypothesized that actin cytoskeletal polymerization induced by hierarchical micro/nanoscale topography is the immediate reason for TAZ activation, while the MAPK signaling pathway is also involved in this process, promoting the activation of TAZ.

In this study, we aimed to investigate the role and activation mechanism of TAZ in topographical cuemediated cell fate decisions. Stable TAZ knockdown periodontal ligament stem cells (PDLSCs) were employed to confirm the role of TAZ, while cytochalasin D and MAPK signaling pathway-specific inhibitors were used to reveal its activation mechanism. Our results demonstrated that TAZ played a key role in this process, and its potential activation mechanism involved actin cytoskeletal polymerization and MAPK signaling pathway activation induced by hierarchical microgroove/nanopore topography.

\section{Materials and Methods}

\section{Specimen Preparation and Surface Characterization}

Selective laser melting combined with alkali heat treatment (SLM-AHT) was employed to prepare titanium 
surfaces with hierarchical microgroove/nanopore topography (experimental group). The key parameters of the selective laser melting system were set as follows: continuous power of $200 \mathrm{~W}$, wavelength of $1054 \mathrm{~nm}$, laser spot size of $0.1 \mathrm{~mm}$, and scanning speed of $7 \mathrm{~m}-\mathrm{s}$. HF $(1.5 \%)$ was used for the following etching procedure, and the samples were then incrementally heated to $600^{\circ} \mathrm{C}$. A smooth titanium surface (Ti) was obtained by sequentially polishing titanium specimens with silicon carbide sandpaper from 240 to 2000 grits (negative control), while sand-blasted, large grit and acid etching titanium surface (SLA) with irregular microscale topography were achieved by sandblasting and acid etching procedures (positive control) as previously described. ${ }^{10}$ The abovementioned titanium specimens were discshaped, $1 \mathrm{~mm}$ in thickness and $10 \mathrm{~mm}$ in diameter (for 48-well plates) or $34 \mathrm{~mm}$ in diameter (for 6-well plates). After preparation, the titanium specimens were cleaned in acetone, absolute ethanol, and double-distilled water $\left(\mathrm{ddH}_{2} \mathrm{O}\right)$ sequentially in an ultrasonic cleaner for 15 minutes, dried at room temperature, and sterilized at a vertical pressure steam sterilizer (SQ810C) for $120^{\circ} \mathrm{C} / 2$ $\mathrm{h}$. The resultant specimens were sterilized with ultraviolet light for 30 minutes before use.

For the surface topography characterization, titanium specimens were observed by a field-emission scanning electron microscope (FE-SEM SUPRA 55 Sapphire, Germany). To obtain low- and high-magnification images of the surface morphology in each group, we observed the images at magnifications of 200 times, 10,000 times and 50,000 times. The 10,000 times images were used to qualitatively evaluate microscale features, and the 50,000 times images were used to evaluate nanoscale features.

\section{PDLSC Isolation and Identification}

Human PDLSCs were isolated according to a previously described protocol, ${ }^{30}$ following approved guidelines by Tianjin Medical University School of Stomatology (Experiment no: TMUhMEC2019011). Patients provided informed consent in accordance with the Declaration of Helsinki. Briefly, the middle one-third periodontal ligament was gently separated from the root surface, digested in a mixed solution of $3 \mathrm{mg} / \mathrm{mL}$ collagenase type I (Sigma, USA) and $4 \mathrm{mg} / \mathrm{mL}$ dispase (Sigma, USA) for $1 \mathrm{~h}$ at $37^{\circ} \mathrm{C}$, and passed through a $70 \mu \mathrm{m}$ strainer (Falcon, USA) to obtain a single-cell suspension. PDLSCs were cultured in fresh $\alpha$-MEM (HyClone, USA) with 10\% FBS (Gibco, USA) and $1 \%$ penicillin/streptomycin (Gibco, USA) and incubated in a $5 \% \mathrm{CO} 2$ atmosphere at $37^{\circ} \mathrm{C}$. Passages $3 \sim 6$ PDLSCs were used for the following experiments. Flow cytometry was employed to identify putative PDLSCs. Generally, $2.5 \times 10^{5} 3$-passage PDLSCs were collected in $1.5 \mathrm{~mL}$ Eppendorf tubes. Then, the cells were washed with PBS 2 times and fixed with 4\% paraformaldehyde for 15 minutes, followed by incubation with FITC-labeled CD45 antibody (eBioscience, USA), FITC-labeled CD90 antibody (eBioscience, USA), and PE-labeled CD146 (eBioscience, USA) for 30 minutes. Finally, cells positively stained with CD90 and CD146 were evaluated with fluorescence-activated cell sorting.

\section{MTS}

For evaluation of the initial cell adhesion on different titanium surfaces, PDLSCs were seeded on specimens in 48 -well plates at a density of $1 \times 10^{4}$ cells/well. After $2 \mathrm{~h}$ of culture, the culture medium was removed, and titanium specimens were gently washed with phosphate-buffered saline (PBS) 3 times. Empty $\alpha$-MEM, MTS, and PMS were added at a ratio of 100:20:1 and incubated at $37^{\circ} \mathrm{C}$ for $4 \mathrm{~h}$. Then, the supernatant was transferred to a new $96-$ well plate to measure the absorbance at $490 \mathrm{~nm}$ wavelength and calculate the OD value.

\section{PDLSC Osteogenic/Adipogenic Induction}

For induction of osteogenic and adipogenic differentiation, PDLSCs were seeded on different titanium specimens in 48 -well plates at a density of $1 \times 10^{5}$ cells/well. The culture medium was changed the next day with osteogenic induction (OI) medium or adipogenic induction (AI) medium and renewed every 3 days. OI medium consisted of $\alpha$ MEM, 10\% FBS, $1 \%$ penicillin/streptomycin, $50 \mu \mathrm{g} / \mathrm{mL}$ ascorbic acid (Sigma, USA), $10 \mathrm{nmol} / 1$ dexamethasone (Sigma, USA) and $5 \mathrm{mmol} / 1 \beta$-glycerophosphate (Sigma, USA). AI medium consisted of $\alpha$-MEM, $10 \%$ FBS, $1 \%$ penicillin/streptomycin, $0.1 \mu \mathrm{M}$ dexamethasone, $1 \mu \mathrm{M}$ insulin (Sigma, USA), $200 \mu \mathrm{M}$ indomethacin (Sigma, USA), and $250 \mu \mathrm{M}$ 3-isobutyl-1-methylxanthine (Sigma, USA). After 3 days of OI, wild-type PDLSCs on different titanium specimens were collected, and qRT-PCR and Western blotting were performed to determine the adhesion-related gene and protein expression levels. Moreover, immunofluorescence staining was employed to visualize the actin cytoskeleton state. After 7 days of OI and AI, alkaline phosphatase (ALP) activity, immunofluorescence staining, qRT-PCR, and Western blotting were employed 
to explore the differentiation of PDLSCs on different titanium surfaces.

\section{ALP Activity Assay}

After 7 days of culture with OI, the culture medium from different groups was collected to evaluate ALP activity using an ALP assay kit (microplate method) (Nanjing Jiancheng, China). The buffer, substrate solution, and color developer were added to the collected culture medium according to the instructions, and the absorbance at $520 \mathrm{~nm}$ was measured with a microplate reader. The experiment was performed in triplicate, and the ALP activity of cells on different topographies was calculated according to the following formula.

ALP activity of culture medium (Jin's unit $/ 100 \mathrm{~mL}$ ) = (Determined OD value - blank OD value)/(standard OD value - blank OD value) $\times$ concentration of phenol standard $(0.02 \mathrm{mg} / \mathrm{mL}) \times 100 \mathrm{~mL} \times$ dilution ratio before sample determination

\section{Establishment of TAZ Knockdown PDLSCs}

Short hairpin RNAs (shRNAs) were used to generate stable TAZ knockdown PDLSCs. TAZ-shRNA1, TAZshRNA2, and TAZ-shRNA3 oligos were purchased from GENEWIZ (China), and the shRNA-scramble plasmid was preserved in our laboratory. shRNA oligos were annealed and ligated into the pLKO.1 vector, producing a final plasmid that expressed the shRNA of TAZ. The recombinant lentiviral vectors were transfected into $293 \mathrm{~T}$ cells and incubated for $12 \mathrm{~h}$ until the medium was replaced with HI ( $\alpha$-MEM, 10\% FBS, $1 \%$ penicillin/streptomycin). The viral supernatants were harvested at 24 and $48 \mathrm{~h}$ and were used to infect PDLSCs at 70\% confluence. After $12 \mathrm{~h}$ of transfection, the cells were cultured for $48 \mathrm{~h}$ with complete medium and then selected with $2 \mu \mathrm{g} / \mathrm{mL}$ puromycin $(1: 1000)$. TAZ knockdown efficiency was measured by qRT-PCR and Western blot experiments as described in more detail in the following sections. High-efficiency TAZ knockdown PDLSCs were selected for subsequent studies. The sequences of the shRNA oligos are shown in Table 1.

For determination of the role of TAZ in topographical cue-induced cell fate determination, TAZ knockdown PDLSCs were seeded on different titanium surfaces, while scramble PDLSCs served as controls. After 3 days of OI culture, the gene expression levels of RUNX2 and PPAR $\gamma$ were detected by qRT-PCR.

\section{qRT-PCR Analysis}

For determination of the effect of hierarchical micro/ nanoscale topography on cell fate decisions, wild-type PDLSCs cultured with OI and AI for 7 days on different titanium specimens were collected to detect osteogenic and adipogenic gene expression levels. Furthermore, for analysis of the role of TAZ in topography-induced cell fate determination, stable TAZ knockdown PDLSCs cultured for 3 days with OI and AI were collected to detect the gene expression levels of RUNX2 and PPAR $\gamma$. Total cellular RNA was extracted using TRIzol reagent (Invitrogen) according to the manufacturer's instructions. qRT-PCR was performed by the ABI Prism 7500 Sequence Amplification System (Applied Biosystems) with the QuantiTect SYBR Green PCR Kit (Qiagen, Hilden, Germany). The housekeeping gene RP0 was used to normalize gene expression. The primers used in this study are listed in Table 2.

\section{Western Blot Analysis}

For evaluation of the effect of different topographies on cell fate decisions, wild-type PDLSCs were collected to determine the total protein expression levels of RUNX2, ALP, and PPAR $\gamma$ after 7 days of culture with OI and AI. For determination of the underlying activation mechanism of TAZ, wild-type PDLSCs were collected to evaluate the protein expression levels of MAPK signaling pathwayrelated proteins, including ERK, p-ERK, JNK, p-JNK,

Table I TAZ shRNA Sequences

\begin{tabular}{|l|l|}
\hline Gene & shRNA Sequences $\mathbf{( 5}^{\prime} \mathbf{- 3} \mathbf{3}^{\prime} \mathbf{)}$ \\
\hline TAZ- & F:5'CCGGGACATGAGATCCATCACTAATCTCGAGATTAGTGATGGATCTCATGTCTTTTTG3' \\
shRNAI & R:5'AATTCAAAAAGACATGAGATCCATCACTAATCTCGAGATTAGTGATGGATCTCATGTC3' \\
TAZ- & F:5'CCGGGGACAAACACCCATGAACATCCTCGAGGATGTTCATGGGTGTTTGTCCTTTTTG3' \\
shRNA2 & R:5'AATTCAAAAAGGACAAACACCCATGAACATCCTCGAGGATGTTCATGGGTGTTTGTCC3' \\
TAZ- & F:5'CCGGCCGTTTCCCTGATTTCCTTGACTCGAGTCAAGGAAATCAGGGAAACGGTTTTTG3' \\
shRNA3 & R:5'AATTCAAAAACCGTTTCCCTGATTTCCTTGACTCGAGTCAAGGAAATCAGGGAAACGG3' \\
\hline
\end{tabular}


Table 2 Primer Sequences for qRT-PCR

\begin{tabular}{|c|c|}
\hline Gene & Primer Sequences (5'-3') \\
\hline RPO & $\begin{array}{l}\text { F: TTCATTGTGGGAGCAGAC } \\
\text { R: CAGCAGTTTCTCCAGAGC }\end{array}$ \\
\hline RUNX2 & $\begin{array}{l}\text { F: AGAAGGCACAGACAGAAGCTTGA } \\
\text { R: AGGAATGCGCCCTAAATCACT }\end{array}$ \\
\hline ALP & $\begin{array}{l}\text { F: CCACGTCTTCACATTTGGTG } \\
\text { R: AGACTGCGCCTGGTAGTTGT }\end{array}$ \\
\hline $\operatorname{PPAR} \gamma$ & $\begin{array}{l}\text { F: CCTTGCAGTGGGGATGTCTC } \\
\text { R: CTTTGGTCAGCGGGAAGGAC }\end{array}$ \\
\hline $\mathrm{C} / \mathrm{EBP} \alpha$ & $\begin{array}{l}\text { F: GGTGGACAAGAACAGCAACGA } \\
\text { R: GTCATTGTCACTGGTCAGCTC }\end{array}$ \\
\hline TAZ & $\begin{array}{l}\text { F: CTGCAGACATCTGCTTCACCA } \\
\text { R: GGGAAGATATGCACCCAGTCC }\end{array}$ \\
\hline CTGF & $\begin{array}{l}\text { F: CAGCATGGACGTTCGTCTG } \\
\text { R: AACCACGGTTTGGTCCTTGG }\end{array}$ \\
\hline CYR6I & $\begin{array}{l}\text { F: GTCCTCCATGGGGTCCTTGA } \\
\text { R: AACTTCATGGTCCCAGTGCTC }\end{array}$ \\
\hline FAK & $\begin{array}{l}\text { F: ACCTCAGCTAGTGACGTATGG } \\
\text { R: CGGAGTCCCAGGACACTGTG }\end{array}$ \\
\hline Integrin $\beta$ I & $\begin{array}{l}\text { F: GCTTTCTGTCACCTCTTCTAATCTTT } \\
\text { R: CGTAGGTGAGAGCTGTTGCATAG }\end{array}$ \\
\hline
\end{tabular}

p38, and p-p38, after 3 days of culture with OI. The collected cells were lysed in RIPA buffer supplemented with PMSF and then centrifuged to collect the supernatant. Following denaturation, the proteins were loaded on 4\%$20 \%$ polyacrylamide gels and then transferred to PVDF membranes. The membranes were incubated for $1 \mathrm{~h}$ to block nonspecific protein binding, incubated with primary antibodies overnight at $4^{\circ} \mathrm{C}$, and incubated with the corresponding secondary antibody for $2 \mathrm{~h}$ the next day. Finally, the membranes were exposed using an ECL kit (CWBIO, Beijing, China) to visualize the immunoreactive protein bands. MAPK pathway antibodies were all purchased from Cell Signaling Technology (USA). RUNX2 and PPAR $\gamma$ antibodies were purchased from Abcam (USA), and ALP antibody was purchased from Abways Technology (Beijing, China).

\section{Pharmacological Inhibitor Treatment}

Pharmacological inhibitors were employed to verify the role of the MAPK signaling pathway in hierarchical micro/ nanoscale topography-induced TAZ activation. After 3 days of culture in 6-well plates on Ti, SLA, and SLM-
AHT surfaces, wild-type PDLSCs were incubated with 10 $\mu \mathrm{M}$ of the ERK inhibitor U0126 (Cell Signaling Technology, USA) and $10 \mu \mathrm{M}$ of the p38 inhibitor SB203580 (Cell Signaling Technology, USA) for $2 \mathrm{~h}$ or $0.2 \mu \mathrm{M}$ cytochalasin D (Abcam, USA) for 10 minutes, and an equivalent volume of DMSO was added as a negative control. After incubation, the cells were harvested, and total RNA was extracted to evaluate the TAZ target gene expression level by qRT-PCR. Moreover, inhibitor-treated PDLSCs on different titanium specimens were tested by immunofluorescence staining to directly reveal TAZ subcellular localization.

\section{Immunofluorescence Staining}

Wild-type PDLSCs cultured on different titanium specimens in 48-well plates were used to visualize the state of the actin cytoskeleton, as well as the RUNX2 expression level. Pharmacological inhibitor-treated PDLSCs were observed to determine the subcellular localization of TAZ. The samples were washed twice with ice-cold PBS, fixed in 4\% paraformaldehyde, permeabilized with $0.05 \%$ Triton X-100 (Sigma-Aldrich), and blocked in BSA $(5 \mathrm{mg} / \mathrm{mL})$. The primary mouse anti-TAZ antibody (1:200, BD) and rabbit anti-RUNX2 antibody (1:200, Abcam) were incubated overnight at $4^{\circ} \mathrm{C}$ and F-actin (1:200, Molecular Probes) was incubated for 30 minutes at room temperature, followed by the fluorescently conjugated secondary antibody (1:200, Invitrogen) incubated at room temperature for $2 \mathrm{~h}$. Finally, the nuclei were stained with DAPI (1:200, Sigma-Aldrich, USA) for 10 minutes. Immunofluorescence images were observed with an inverted fluorescence microscope system (FluoView FV1000; Olympus).

\section{Statistical Analysis}

All experiments were repeated at least 3 times to ensure the accuracy and reliability of the data, and data analysis was carried out with IBM SPSS statistics 23 software. One-way ANOVA was used to compare multiple samples. $\mathrm{P}<0.05$ was considered significant, and $\mathrm{P}<0.01$ was considered highly significant $\left(* \mathrm{P}<0.05, * * \mathrm{P}<0.01,{ }^{* * *} \mathrm{P}<0.001\right)$.

\section{Results and Discussion}

\section{Surface Topography Characterization and PDLSC Identification}

In our published study, the DMLS titanium surface with hierarchical microprotrusion/nanonotch topography exhibited a 
greater potential for promoting BMSC osteogenic differentiation both in vitro and in vivo than the Ti and SLA surfaces. ${ }^{10}$ In the present study, we upgraded the fabrication method by the combined use of the selective laser melting technique and alkali heat treatment to obtain an SLM-AHT surface with hierarchical microgroove/nanopore topography without apparent microparticles. FE-SEM was employed to observe the titanium surfaces, and the images are presented in Figure 1A. The Ti surface possessed no apparent micro- or nanostructures. The SLA surface presented a rough structure on the microscale without regular features on the nanoscale. By contrast, the SLM-AHT surface exhibited a micron-sized groove with an average diameter of $30-40 \mu \mathrm{m}$ combined with nanosized mesh-like pores with a diameter of approximately $100 \mathrm{~nm}$.

PDLSCs are a group of MSC-like populations with multidifferentiation potential. ${ }^{30,31}$ Herein, our flow cytometric analysis of PDLSCs showed positive expression of the typical mesenchymal cell surface markers CD90 and CD146 and negative results for the hematopoietic cell marker CD45 (Figure 1B). Putative PDLSCs were employed in the following experiments.

\section{PDLSC Adhesion and Actin Cytoskeleton State on Different Titanium Surfaces}

Generally, the implant surface could be considered the cellular microenvironment to direct cell behavior. ${ }^{32}$ Cells initially respond to their microenvironment by integrin-mediated adhesion. ${ }^{2,33,34}$ The alteration in cell adhesion could translate physical cues into biochemical signaling and eventually affect the cell phenotype; for example, the formation of focal adhesion could activate FAK and paxillin, ${ }^{35} \mathrm{MAPK},{ }^{36}$ and Rho, ${ }^{37}$ which are involved in regulation of stem cell

\section{A}
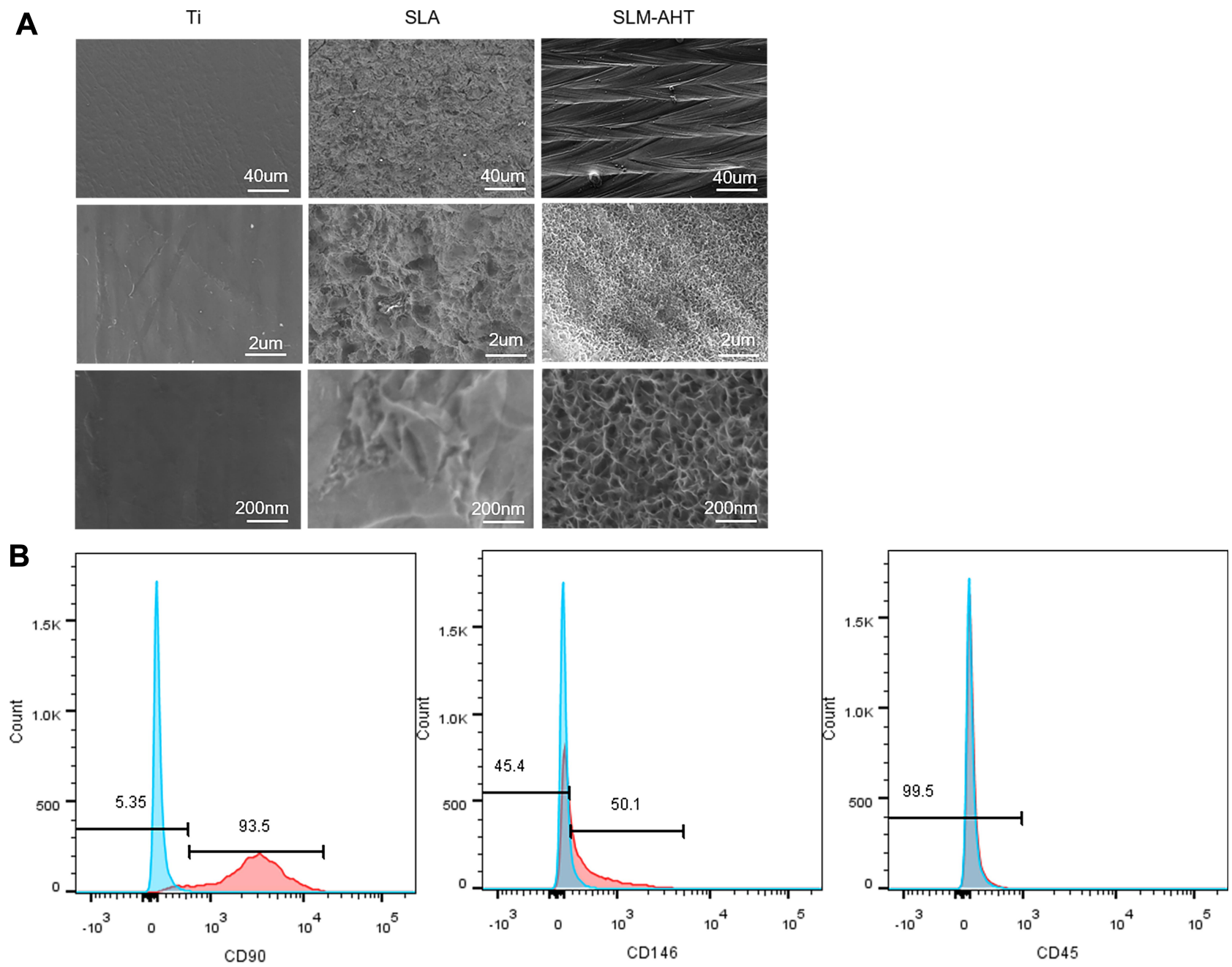

Figure I Surface characterization of Ti, SLA and SLM-AHT titanium surfaces and flow cytometry assay for PDLSCs identification. (A)FE-SEM observation of Ti, SLA and SLM-AHT titanium surface at 200x, 10,000× and 50,000× magnification. (B) Flow cytometric analysis of PDLSCs showed positive expression of cell markers CD90, CDI46 and negative results for CD45. 
lineage differentiation. ${ }^{38,39}$ Therefore, in the present study, the adhesion process was initially assayed to investigate the effect of hierarchical micro/nanoscale topography (SLMAHT surface) on cell behavior. The MTS results are displayed in Figure 2A. More cells were observed on the SLM-AHT surface than the other surfaces, suggesting that the SLM-AHT surface could promote cell adhesion. Then, we detected the gene expression levels of the adhesionrelated genes integrin $\beta 1$ and FAK after 3 days of culture, and integrin $\beta 1$ and FAK expression was significantly increased on the SLM-AHT surface compared with the Ti and SLA surfaces with or without OI (Figure 2B). Moreover, Western blot results further demonstrated that the total protein levels of integrin $\beta 1$ and vinculin in the SLM-AHT group were higher than those in the other two groups with or without OI (Figure 2C), indicating that the SLM-AHT surface could promote cell adhesion by increasing the expression levels of adhesion-related proteins. Since the cytoskeleton connected to adhesion-related proteins could conduct and amplify topographical cues and was considered necessary in the topography-elicited cell fate decision, ${ }^{40,41}$ we next evaluated the state of the actin cytoskeleton on hierarchical microgroove/nanopore topography. As shown by the immunofluorescence results, the PDLSCs cultured on the SLM-AHT surface indeed had more prominent stress fibers than the cells cultured on the Ti and SLA surfaces (Figure 2D), verifying that the SLM-AHT surface could promote cytoskeletal reorganization. Thus, the SLM-AHT surface with hierarchical micro/nanoscale topography was more favorable for cell adhesion and actin cytoskeletal polymerization than the Ti and SLA surfaces, which was consistent with previous studies. ${ }^{10,20}$ However, further exploration is required to determine the role and molecular mechanism of cell adhesion and the actin cytoskeleton in topographical cueinduced cell fate determination.

\section{Osteogenic and Adipogenic Differentiation of PDLSCs on Different Titanium Surfaces}

In our experiments, PDLSCs were employed to investigate the role of the hierarchical micro/nanoscale topography on cell fate
A

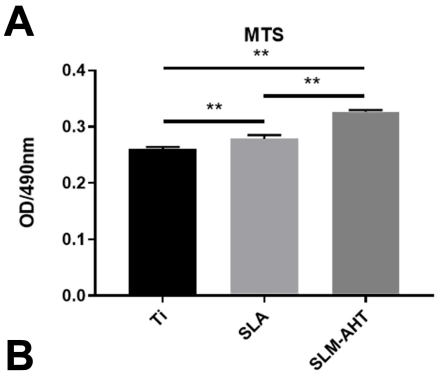

C
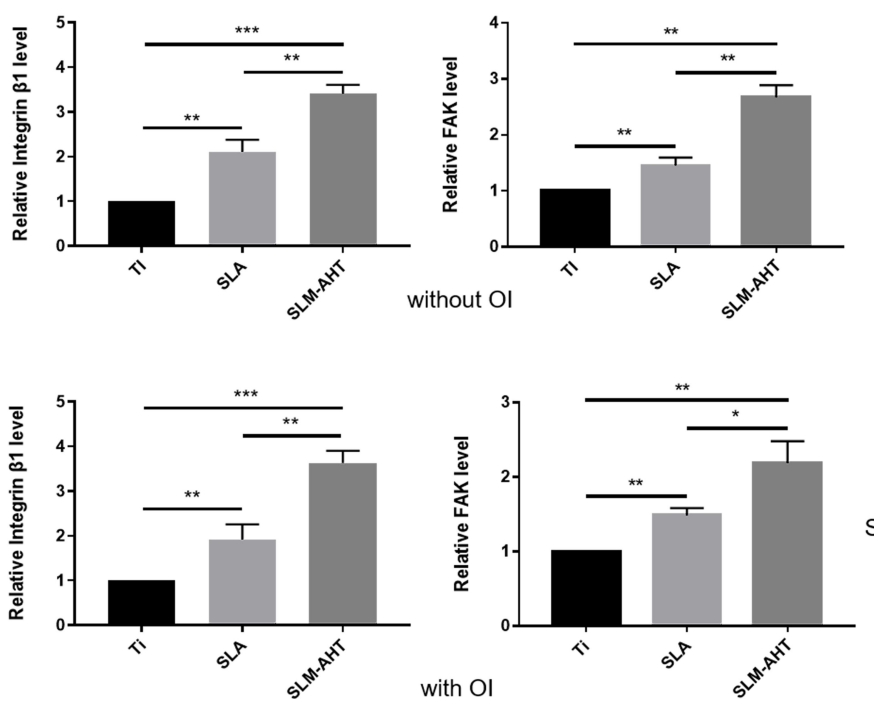

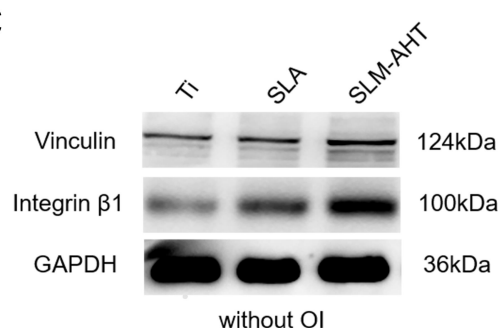

D

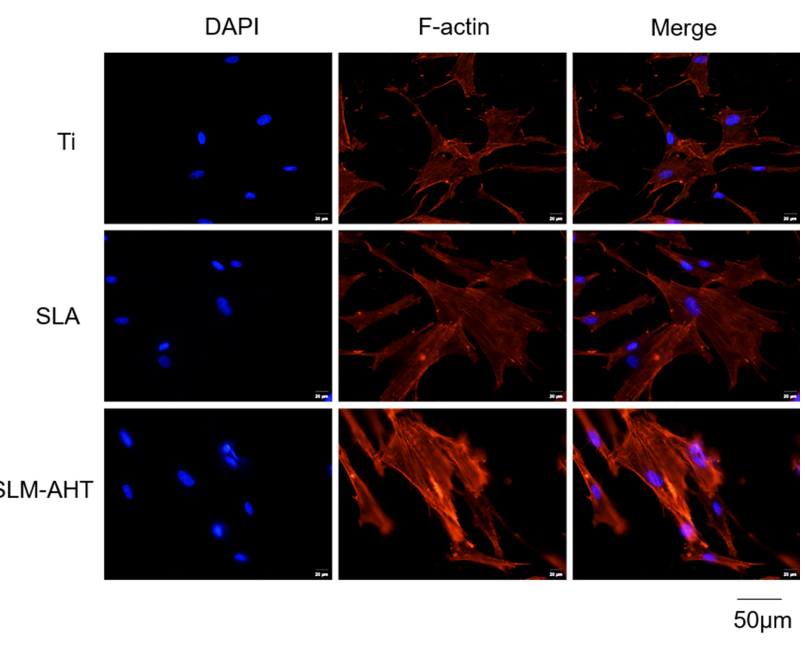

Figure 2 PDLSCs adhesion and actin cytoskeletal state on different titanium surfaces. (A) MTS assays for cell early adhesion on different titanium surfaces after 2 hours of culture. (B) The relative mRNA levels of integrin $\beta I$ and FAK in PDLSCs cultured on Ti, SLA and SLM-AHT surfaces for 3 days with and without osteoinduction. (C) The total protein expression of integrin $\beta I$ and vinculin in PDLSCs cultured on different surfaces for 3 days with and without osteoinduction. (D) Immunofluorescence staining assays for actin cytoskeleton state of PDLSCs cultured on different surfaces for 3 days (red, F-actin; blue, DAPI). Error bars represent SD ( $n=3)$. $* \mathrm{P}<0.05$, $* * P<0.0 \mathrm{I}$, $* * * \mathrm{P}<0.00 \mathrm{I}$. 
A
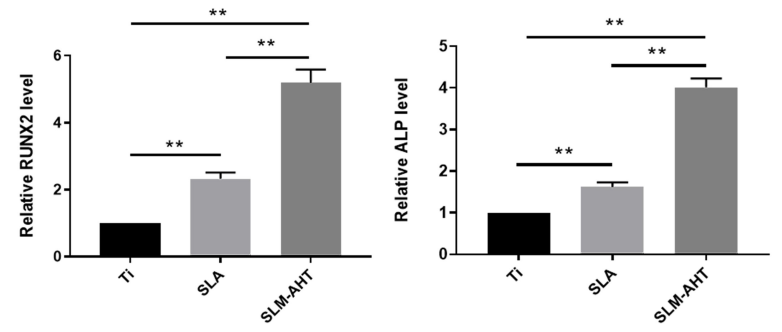

B DAPI
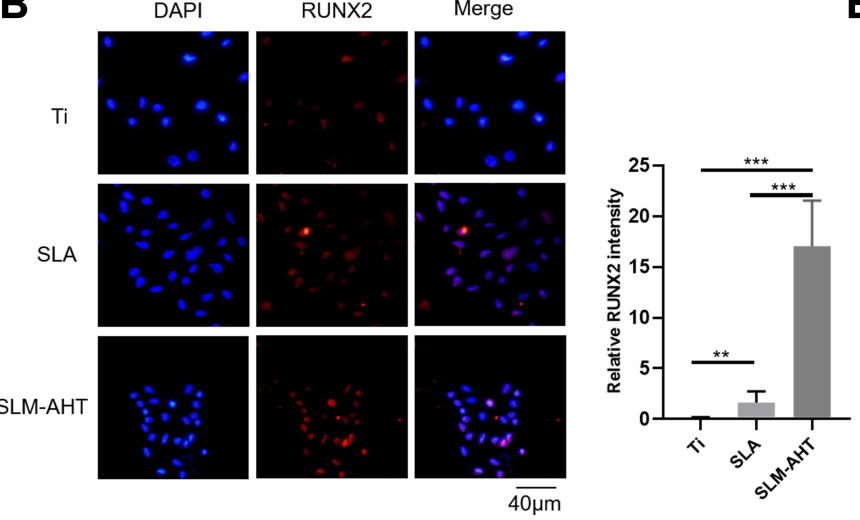

SLM-AHT

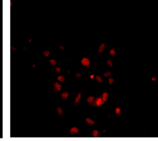

$40 \mu \mathrm{m}$

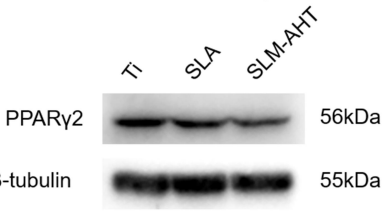

C

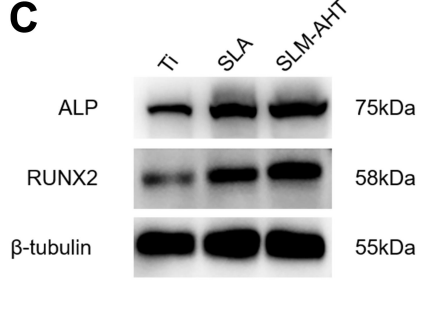

E

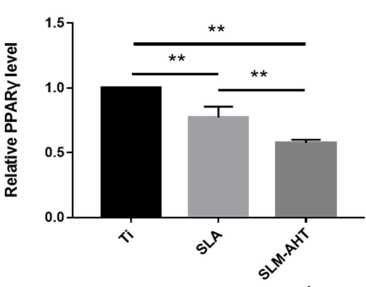

$\mathbf{F}$
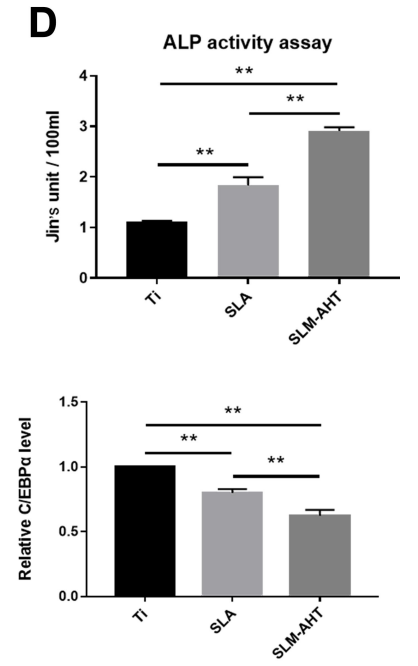

Figure 3 Osteogenic and adipogenic differentiation of PDLSCs on different titanium surfaces. (A) The relative mRNA levels of RUNX2 and ALP in PDLSCs cultured on different surfaces for 7 days with osteoinduction. (B) (Left panel) Immunofluorescence staining assay for RUNX2 in PDLSCs (red, RUNX2; blue, DAPI) cultured on different surfaces for 7 days. (Right panel) Immunofluorescence stain intensity analysis of RUNX2. (C) Total protein expression of RUNX2 and ALP in PDLSCs at 7 days after osteoinduction. (D) ALP activity of PDLSCs cultured on Ti, SLA, and SLM-AHT titanium surfaces for 7 days. (E) The relative mRNA levels of PPAR $\gamma$ and C/EBP $\alpha$ in PDLSCs cultured in the adipogenic conditions on different titanium surfaces for 7 days. (F) The total protein expression of PPAR $\gamma$ in PDLSCs at 7 days after adipoinduction was examined by Western blot. Error bars represent $\mathrm{SD}(\mathrm{n}=3)$. **P $<0.01$, ***P $<0.001$.

decision. After 7 days of osteogenic induction, the SLM-AHT group exhibited significantly higher gene expression levels of the osteogenic markers RUNX2 and ALP than the Ti and SLA groups (Figure 3A). Immunofluorescence data showed that SLM-AHT group had the strongest RUNX2-positive staining among the groups (Figure 3B). The total protein expression levels of RUNX2 and ALP in the SLM-AHT group were higher than those in the Ti and SLA groups (Figure 3C). In addition, enhanced ALP activity was observed on the SLMAHT surface $(\mathrm{P}<0.05)$ (Figure 3D). Taken together, these data indicated that the microgroove/nanopore topography we fabricated could promote PDLSC osteogenic differentiation, which was consistent with previous studies. ${ }^{6,7}$ Moreover, the expression levels of the adipogenic genes PPAR $\gamma$ and $\mathrm{C} / \mathrm{EBP} \alpha$ were analyzed after 7 days of adipogenic induction. As shown in Figure 3E, the SLM-AHT group had the lowest expression levels among all three groups. Accordingly, the lowest PPAR $\gamma 2$ protein expression was also found with the SLMAHT surface (Figure 3F), indicating that the hierarchical micro/nanoscale topography could inhibit adipogenic differentiation of PDLSCs.

Long-term success of intraosseous implants requires a microenvironment that could promote osteogenic differentiation of stem cells as well as inhibit adipogenic differentiation. ${ }^{32}$ Thus, the effect of different surface topographies on cell differentiation has been intensively studied in recent years. It was reported that hierarchical micro/nanoscale topography has a substantial advantage for bone formation over individual microscale or nanoscale topography because of its similar scale features to the natural bone structure. ${ }^{6,8,42}$ Our previous study demonstrated that the SLM-AHT surface with hierarchical microgroove/nanopore topography could promote preosteoblast MC3T3-E1 cells osteogenic differentiation by enhancing integrin $\alpha 2$-related cell adhesion. ${ }^{43}$ In the current study, we revealed that the SLM-AHT surface could not only enhance osteogenic differentiation of PDLSCs but also inhibit adipogenic differentiation. Nevertheless, the mechanotransduction mechanism of SLM-AHT surface directing cell fate remains to be elucidated.

\section{TAZ Activation is Essential in Topography-Mediated Regulation of PDLSC Differentiation}

YAP/TAZ are core molecules of the Hippo signaling pathway that mediate widespread biological processes. ${ }^{44}$ 
Increasing evidence has shown that TAZ could respond to mechanical cues and ultimately be involved in stem cell fate decision. As a transcriptional coactivator, TAZ requires nuclear translocation for its transcriptional activity. ${ }^{13}$ Accordingly, the subcellular localization of TAZ and its transcriptional activity were successively detected to determine whether TAZ activation occurs when cells perceive hierarchical micro/nanoscale topography. We performed immunofluorescence and determine the TAZ localization to evaluate the distribution of TAZ on three different titanium surfaces. As expected, increased TAZ nuclear localization was observed on the SLM-AHT surface compared with the SLA surfaces (Figure 4A), indicating hierarchical microgroove/nanopore topography could promote nuclear translocation of TAZ. Connective tissue growth factor (CTGF) and cysteine-rich angiogenic inducer 61 (Cyr61) are well-characterized TAZ target genes located in the nuclei. After nuclear translocation, TAZ binds to and predominantly activates the TEA domain DNA-binding transcription factors (TEAD) 1-4, thereby stimulating the expression of multiple genes, including CTGF and CYR61. These genes are commonly used as indicators of TAZ transcriptional activation in many published papers. ${ }^{45}$ Thus, the expression levels of TAZ and its target genes CTGF and CYR61 were detected by qRT-PCR to confirm the transcriptional activity of TAZ. Interestingly, the TAZ gene expression of the SLM-AHT group had no significant difference with those
A

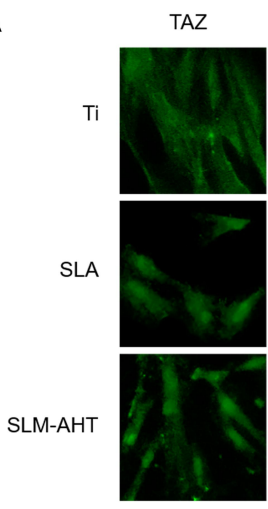

B

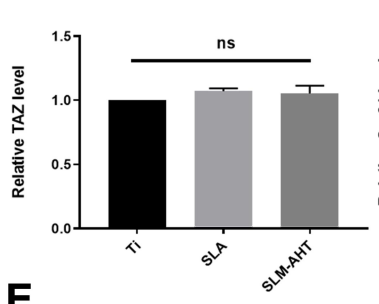

E

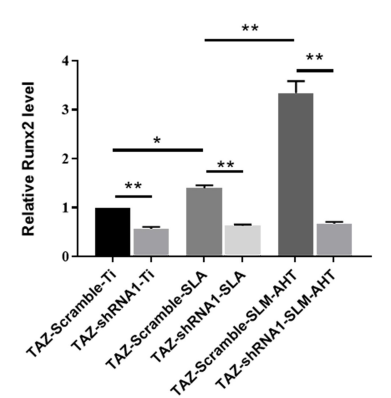

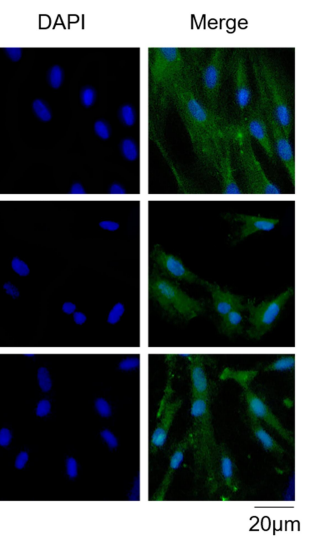
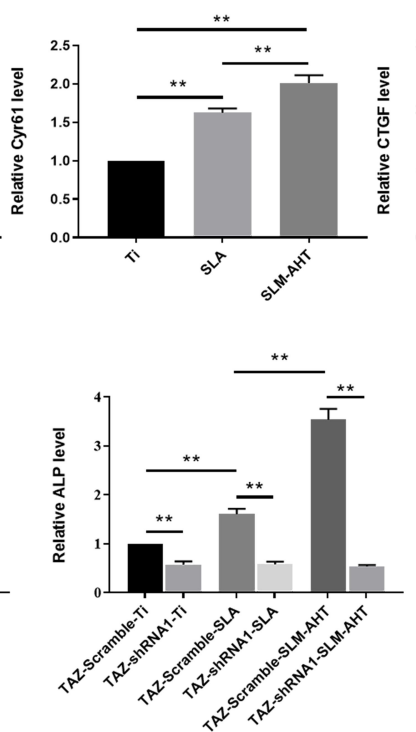

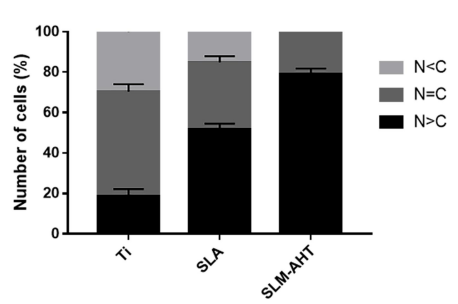

C

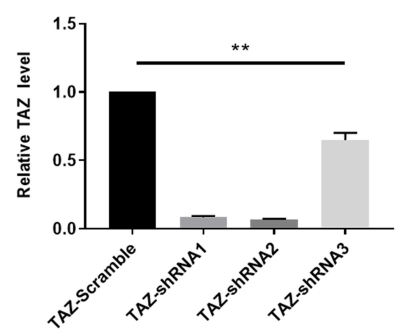

D
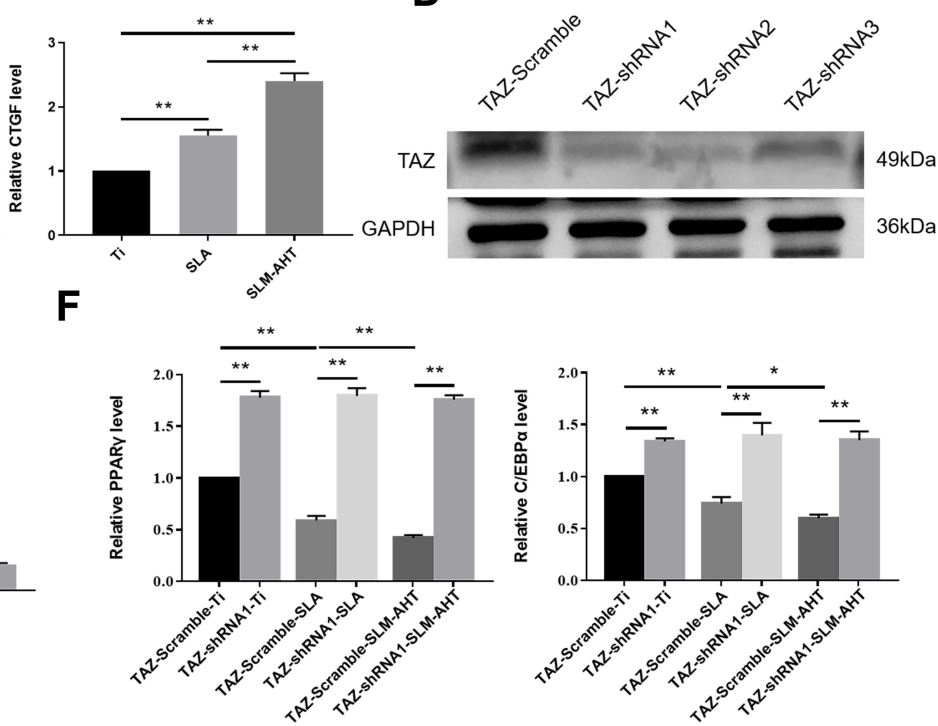

Figure 4 The role of TAZ on hierarchical micro/nanoscale topography directing cell fate. (A) (Left panel) Immunofluorescence staining assays for TAZ subcellular localization in PDLSCs osteoinduction cultured on different titanium surfaces for 3 days (green, TAZ; blue, DAPI). (Right panel) The quantification of TAZ subcellular localization ( $N$, nuclear; $\mathrm{C}$, cytoplasmic). (B) qRT-PCR analysis of the relative mRNA level of TAZ and TAZ target genes including CTGF and Cyr6I in PDLSCs osteoinduction cultured on different titanium surfaces for 3 days. Error bars represent SD $(n=3)$. ${ }^{*} P<0.05$. ${ }^{* * P}<0.01$. (C) The relative mRNA levels of TAZ in stable TAZ knockdown PDLSCs. (D) Protein expression of TAZ in stable TAZ knockdown PDLSCs. (E) The relative mRNA levels of RUNX2 and ALP in stable TAZ knockdown PDLSCs cultured in the osteogenic conditions for 3 days. (F) The relative mRNA levels of PPAR $\gamma$ and C/EBP $\alpha$ in stable TAZ knockdown PDLSCs cultured in the adipogenic conditions for 3 days. 
of the other two groups, but the CTGF and Cyr61 expression levels were significantly increased in the SLM-AHT group (Figure 4B), suggesting hierarchical microgroove/ nanopore topography could improve transcriptional activity of TAZ without significantly promoting its mRNA expression. Collectively, our data demonstrated that microgroove/nanopore topography was sufficient to facilitate TAZ activation by increasing its nuclear localization as well as enhancing its transcriptional activity.

Next, we explored the functional role of TAZ activation in topography-induced cell fate decision by loss-offunction experiments. To this end, we generated TAZ knockdown PDLSCs by three shRNAs, and two of them were confirmed to be effective by qRT-PCR and Western blots (Figure 4C, D). We randomly chose TAZ-shRNA1 PDLSCs to perform the following experiments, while PDLSCs transinfected with scramble shRNA served as controls. The expression levels of the osteogenesis-related genes RUNX2 and ALP as well as the adipogenesisrelated genes PPAR $\gamma$ and $\mathrm{C} / \mathrm{EBP} \alpha$ were detected. As shown in Figure 4E and $\mathrm{F}$, the cells transinfected with scramble shRNA exhibited significantly increased gene expression levels of RUNX2 and ALP and decreased gene expression levels of PPAR $\gamma$ and C/EBP $\alpha$ on the SLM-AHT surface compared with Ti and SLA surfaces, similar to the wild-type PDLSCs. In contrast, the TAZ knockdown cells showed no significant differences in both the osteogenesis-related and adipogenesis-related gene expression levels among the three titanium surfaces, and the TAZ knockdown cells showed lower osteogenesisrelated gene expression levels and higher adipogenesisrelated gene expression levels compared with the cells transinfected with scramble shRNA, indicating that TAZ was essential in hierarchical microgroove/nanopore topography-induced stem cell fate decision.

As a transcriptional coactivator, TAZ contains a WW domain that can bind to Pro-Pro-X-Tyr motifs of the osteogenic transcription factor RUNX2 and the adipogenic transcription factor PPAR $\gamma$ and is ultimately involved in stem cell fate determination. ${ }^{18,46,47} \mathrm{YAP} / \mathrm{TAZ}$ can act as downstream elements in cell detection of mechanical microenvironment, such as ECM rigidity, mechanical stress, or strain. ${ }^{12,48,49}$ For intraosseous implants, the surface features could be recognized as microenvironment cues to modulate YAP/TAZ activity and play a vital role in changes in cell behavior, especially promotion of osteogenic differentiation. Nanotube surfaces were reported to activate TAZ by increasing its nuclear localization, ${ }^{19}$ and
YAP-mediated mechanotransduction was recently revealed as essential in hierarchical macropore/nanowire structureinduced BMSC osteogenic differentiation. ${ }^{20}$ In this study, we proved that the SLM-AHT surface with microgroove/ nanopore topography could facilitate TAZ activation by promoting nuclear localization and transcriptional activity of TAZ, while deletion of TAZ resulted in the SLM-AHT surface failing to promote PDLSC osteogenic differentiation and inhibit adipogenic differentiation. In conclusion, TAZ plays a prominent role in hierarchical microgroove/ nanopore topography regulation of PDLSC lineage decisions, and the underlying mechanism of TAZ activation when cells perceive the SLM-AHT surface was further investigated in the subsequent experiments.

\section{Hierarchical Micro/Nanoscale Topography-Induced Actin Cytoskeleton Polymerization is Required for TAZ Activation}

The polymerization of the actin cytoskeleton was shown to be required to maintain TAZ nuclear localization. ${ }^{12,50,51}$ Given that mechanotransductive regulation is closely related to the integrity of the actin cytoskeleton ${ }^{52}$ and that we have already proved that the SLM-AHT surface could induce actin polymerization in this work, we hypothesized that actin cytoskeletal polymerization triggered by hierarchical micro/nanoscale titanium surface was essential for TAZ activation. To verify this theory, we used cytochalasin D to inhibit polymerization of the actin cytoskeleton. ${ }^{53}$ As shown by the immunofluorescence results and statistical analysis of the percentage of nuclear TAZ intensity against total TAZ intensity, TAZ was predominantly localized in the cytoplasm in all cytochalasin D-treated groups, and were no longer detected a visible difference in TAZ subcellular localization among the different titanium surfaces. By contrast, in the DMSOtreated negative control groups, the subcellular distribution of TAZ was similar to that of the wild-type cells, exhibiting an obviously increased nuclear localization on the SLM-AHT surface compared with the Ti and SLA surfaces (Figure 5A, B). Therefore, actin cytoskeletal polymerization triggered by the hierarchical micro/nanoscale titanium surface could be considered indispensable for TAZ nuclear localization. Furthermore, qRT-PCR showed that in the cytochalasin D-treated groups, we no longer detected a significant difference in the expression levels of the TAZ target genes Cyr61 and CTGF among the three different 
A

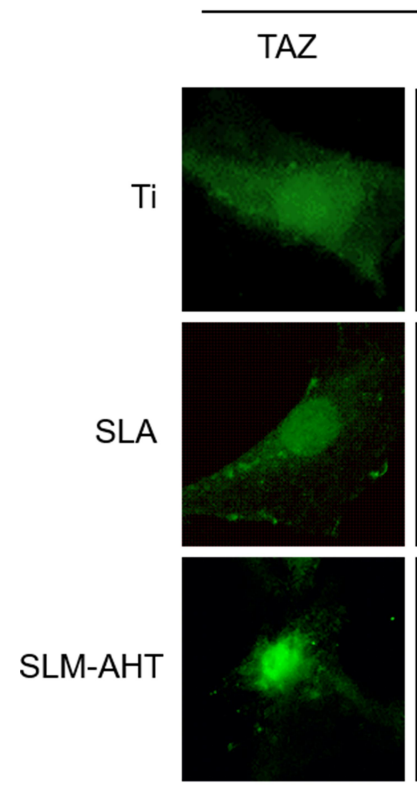

B

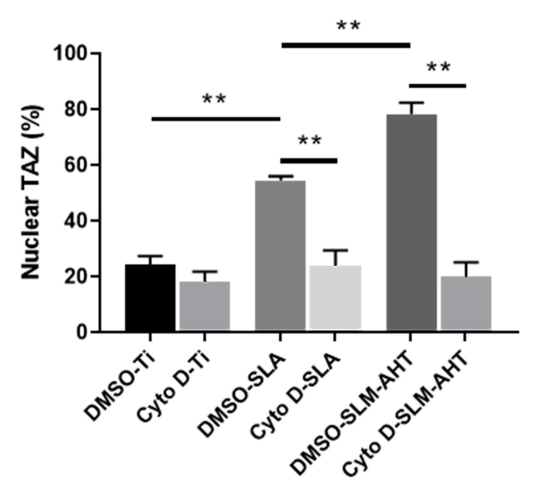

DMSO

DAPI
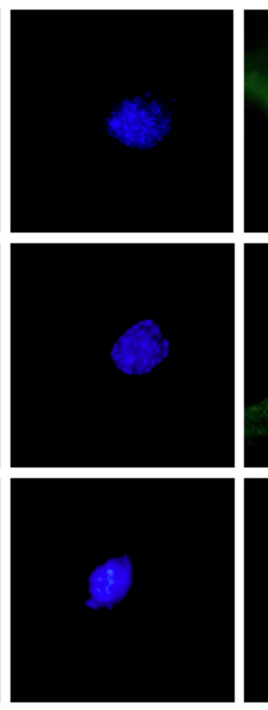

C
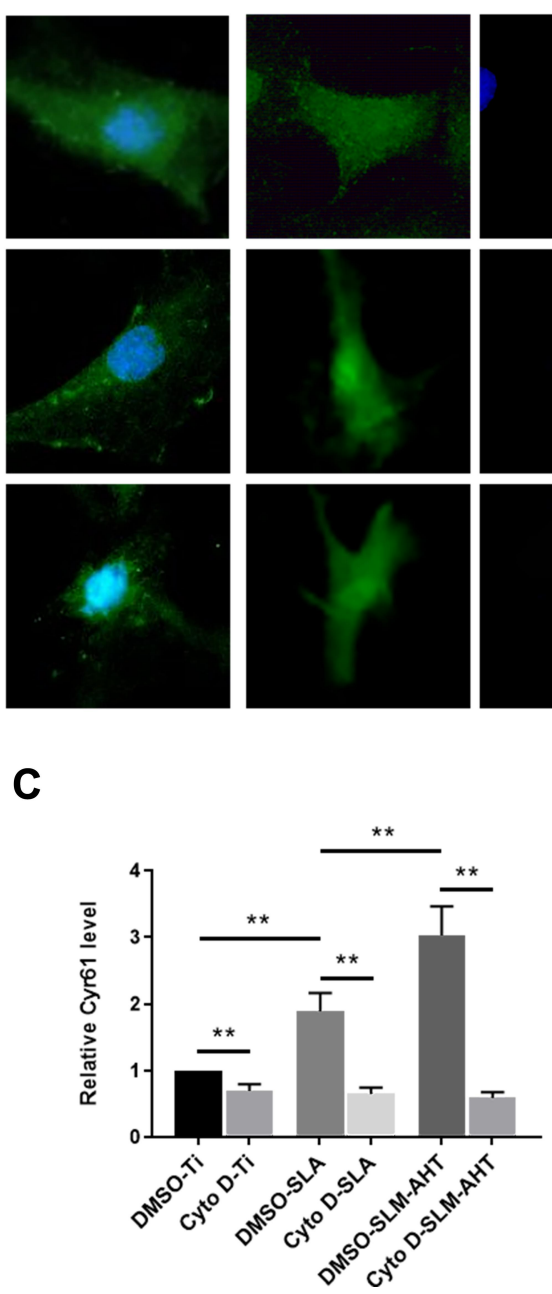

Cytochalasin D

\begin{tabular}{lll}
\hline TAZ & DAPI & Merge
\end{tabular}
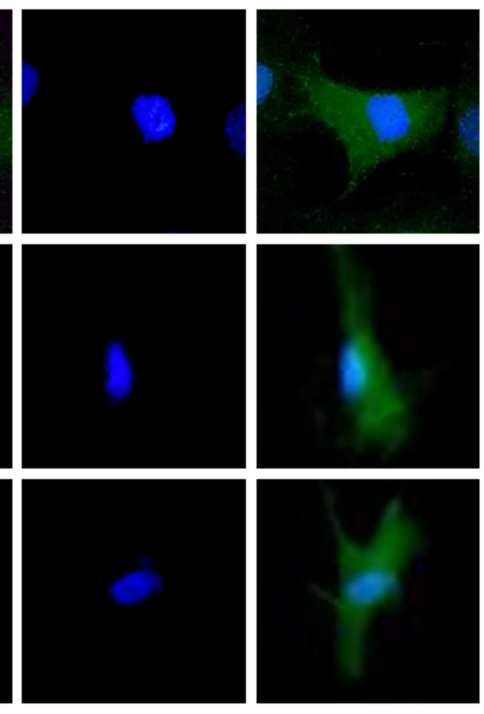

$\overline{10 \mu \mathrm{m}}$

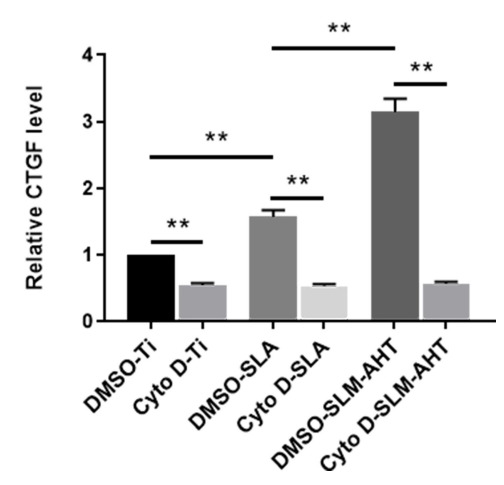

Figure 5 Hierarchical micro/nanoscale topography-induced actin cytoskeleton polymerization is required for TAZ activation. (A) Immunofluorescence staining for TAZ in Cytochalasin D treated PDLSCs cultured on Ti, SLA and SLM-AHT titanium surfaces after 3 days osteoinduction (green, TAZ; blue, DAPI). (B) The statistical analysis of the percentage of nuclear TAZ intensity against total TAZ intensity of $(\mathbf{A})$. (C) The relative mRNA levels of TAZ target genes Cyr6I and CTGF in cytochalasin D treated PDLSCs on different titanium surfaces after 3 days osteoinduction. Error bars represent SD $(n=3)$. **P $<0.01$.

titanium surfaces (Figure 5C), suggesting that the inhibition of actin cytoskeletal polymerization could ultimately disturb transcriptional activity of TAZ promoted by hierarchical micro/nanoscale topography. Collectively, the results indicated that polymerization of the actin cytoskeleton in response to hierarchical micro/nanoscale topography was essential to TAZ activation.

TAZ was initially defined as downstream molecule of the Hippo signaling pathway and was subsequently identified as a downstream molecule of mechanical cues. However, the relationship among the Hippo signaling pathway, mechanical cues, and TAZ has not been fully elucidated. TAZ activation on stiff ECM required cytoskeletal tension and was independent of the Hippo signaling pathway. ${ }^{12}$ However, another study reported that the cytoskeleton could carry out its biological function in a Hippo signaling pathway-dependent way. For instance, stress fibers, serving as the main mechanical cues modulator, could regulate the Hippo signaling pathway by changing the cell morphology and could subsequently modulate cell proliferation. ${ }^{51}$ Moreover, F-actin regulator proteins could affect the Hippo signaling pathway and ultimately be involved in cell growth regulation. ${ }^{50}$ Overall, the findings suggested that hierarchical micro/nanoscale topographytriggered cytoskeletal reorganization may lead to TAZ activation through the Hippo signaling pathway, and the 
regulatory network among the cytoskeleton, TAZ, and the Hippo signaling pathway needs to be further investigated in the future. In addition, the cytoskeleton physically connects focal adhesions on the cell surface to the cell nucleus and passes mechanical cues to the nucleus, resulting in chromosomal arrangement and gene transcriptional activation or repression, ${ }^{33,54,55}$ suggesting the existence of epigenetic regulation. In our previous study, we indeed proved that topographical cues could promote the osteogenic differentiation of BMSCs by inducing a rapid H3K27 demethylation and increased the level of $\mathrm{H} 3 \mathrm{~K} 4 \mathrm{me} 3$ at the promoter of the RUNX2 gene. ${ }^{10}$ From this aspect, the role of cytoskeleton in TAZ activation as well as the underlying epigenetic mechanism also deserve further systematic exploration.

\section{Hierarchical Micro/Nanoscale Topography Activation of the MAPK Signaling Pathway is Necessary for TAZ Activation}

Strong evidence has shown that the MAPK signaling pathway could respond to mechanical cues and be involved in cell fate decision. ${ }^{26,28}$ Moreover, single nanoscale topography could activate MAPK signaling pathway. ${ }^{19}$ Therefore, we hypothesized that the regulation of cell fate determination by the SLM-AHT surface with hierarchical micro/nanoscale topography was mediated by MAPK activation. Since ERK, JNK and p38 were identified as three key molecules of MAPK signaling pathway, ${ }^{56}$ we examined both total and phosphorylated protein expression levels of these three molecules on different titanium surfaces to determine whether the MAPK signaling pathway was involved in hierarchical micro/nanoscale topography-mediated cell fate decision. As shown in Figure 6A, compared with those of the control groups, the protein expression levels of ERK1/2, p38, JNK, and p-JNK in the SLM-AHT group did not exhibit significant differences, whereas the p-ERK1/2 and p-p38 expression levels were significantly increased. The results suggested that the micro/nanoscale topography could activate the ERK and p38 MAPK signaling pathway. Interestingly, it was reported that a nanotopographical plate could active the ERK and JNK MAPK signaling pathway. ${ }^{19}$ The conflicting results possibly reflected scale-specific responses
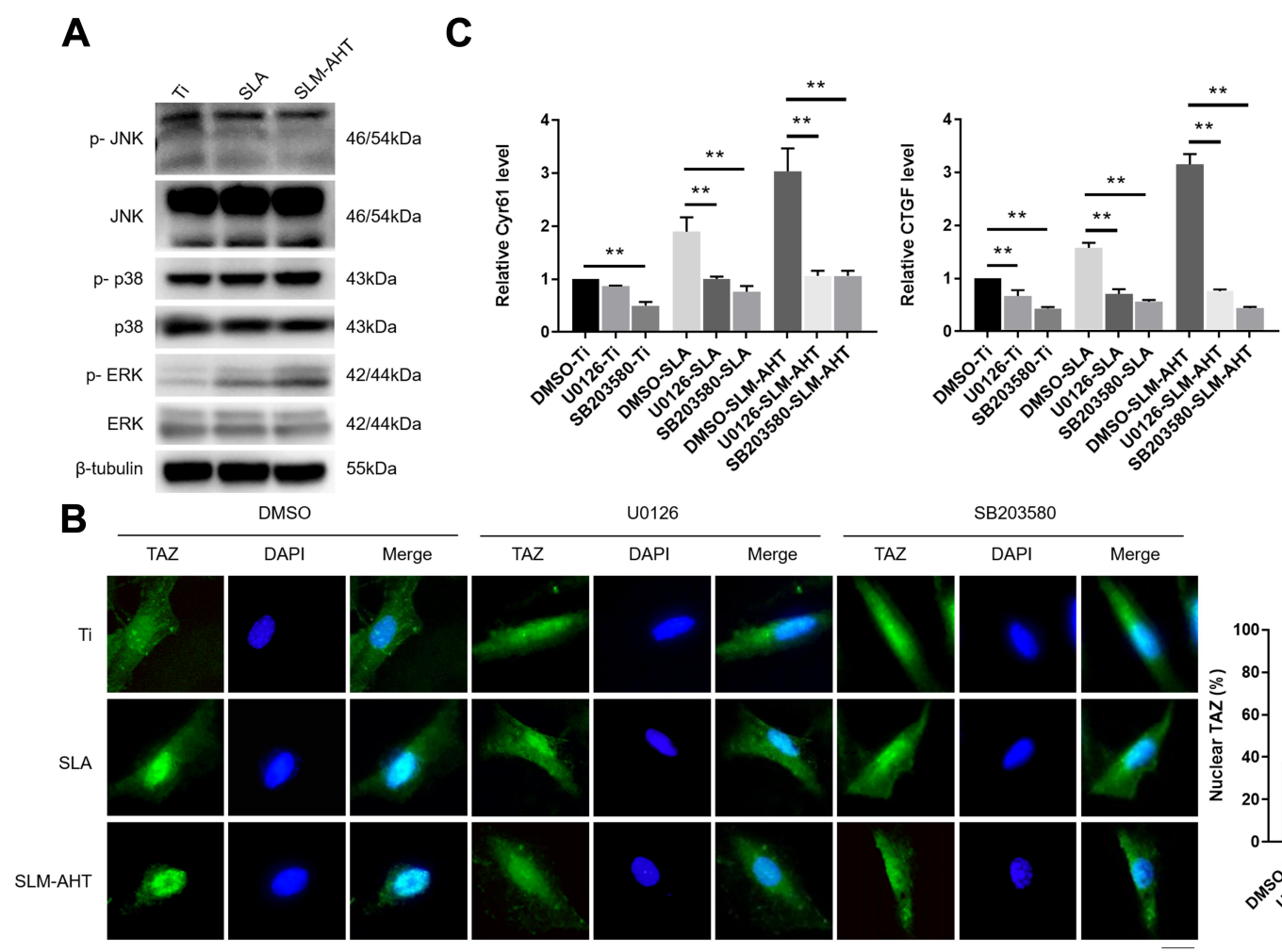
SB203580

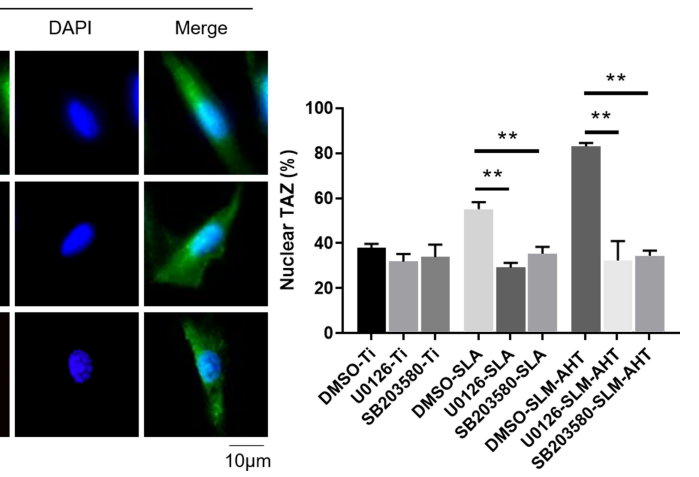

Figure 6 Hierarchical micro/nanoscale topography activating MAPK signaling pathways is essential for TAZ activation. (A) The protein expression levels of MAPK signaling pathways molecules by Western blot after 3 days osteoinduction on Ti, SLA and SLM-AHT surfaces. (B) (Left panel) Immunofluorescence staining for TAZ in U0I26 or SB203580-treated PDLSCs cultured on Ti, SLA and SLM-AHT titanium surfaces after 3 days osteoinduction (green, TAZ; blue, DAPI). (Right panel) The statistical analysis of the percentage of nuclear TAZ intensity against total TAZ intensity. (C) The relative mRNA levels of TAZ target genes Cyr6I and CTGF in U0I26 or SB203580-treated PDLSCs on different titanium surfaces after 3 days osteoinduction. Error bars represent $S D(n=3)$. **P <0.0I. 
to the topographical features, indicating a requirement for reproducible manufacture to ensure the stable size of implant surface features. ${ }^{57}$

Based on the fact that mechanical cue-induced MAPK signaling pathway activation could promote nuclear localization of $\mathrm{TAZ}^{58}$ and that hierarchical micro/nanoscale topography could enhance TAZ nuclear translocation, we investigated whether topographical cue-induced TAZ nuclear localization was associated with MAPK signaling pathway. To this end, the ERK inhibitor U0126 and the p38 inhibitor SB203580 were employed for the following experiments, with DMSO serving as a blank control. The results showed that the cells in the control group exhibited increased TAZ with unclear localization on the SLM-AHT surface compared to the other two surfaces, while both the
U0126-treated cells and the SB203580-treated cells showed no significant difference in TAZ subcellular localization among the three titanium surfaces (Figure 6B), indicating that the ERK and p38 MAPK signaling pathways were both necessary for TAZ nuclear translocation. Additionally, transcriptional activity of TAZ was determined to further verify the role of the MAPK signaling pathway in TAZ activation. As shown in Figure 6C, with DMSO treatment, increasing gene expression levels of CTGF and CYR61 in the SLM-AHT group were observed. In contrast, the U0126- and SB203580-treated groups exhibited similar expression levels of CTGF and CYR61 on the three different titanium surfaces, suggesting that the ERK and p38 MAPK signaling pathways were both indispensable to enhance TAZ transcriptional activity. The

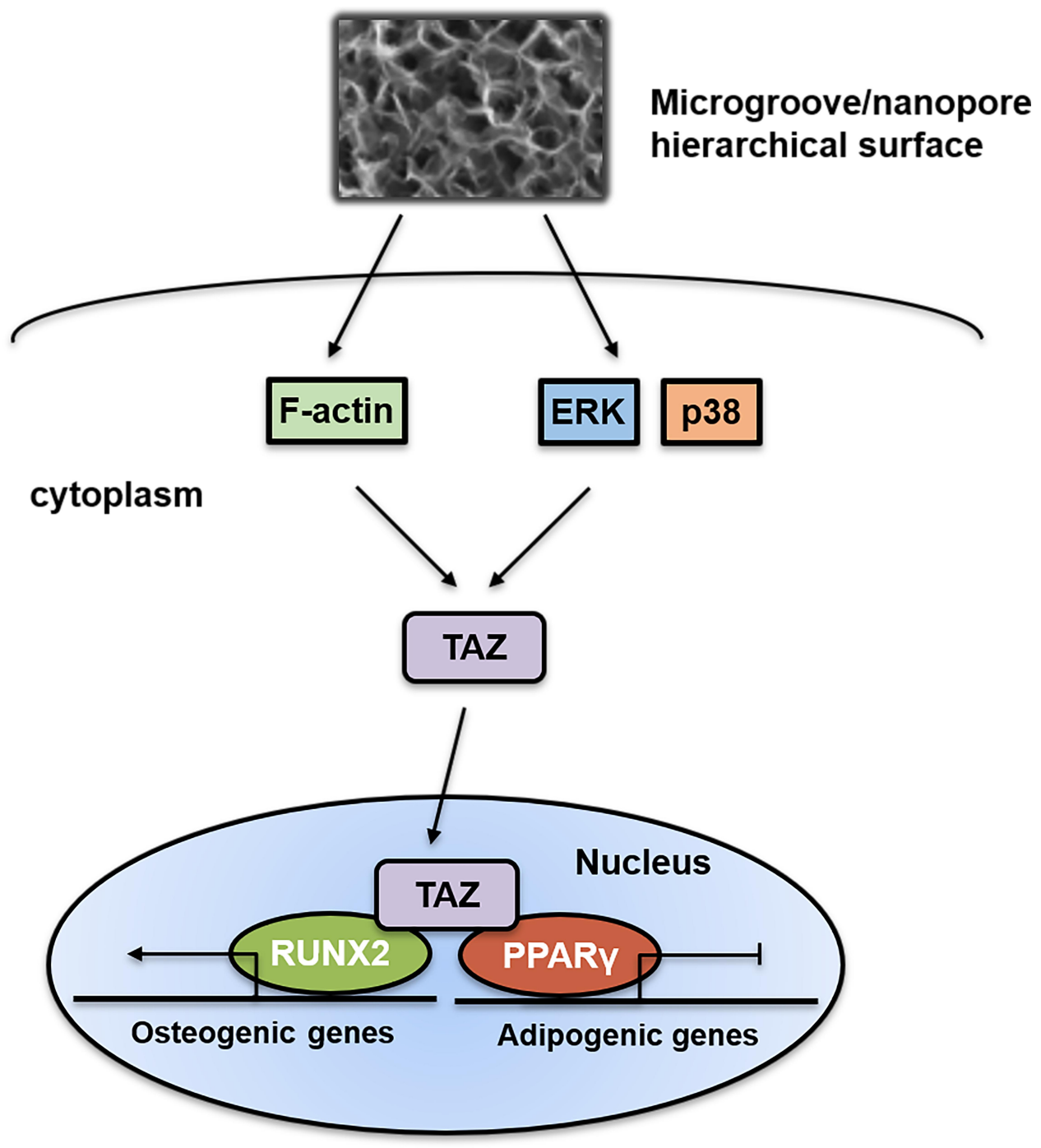

Figure 7 Schematic representation of the role and activation mechanism of TAZ in hierarchical microgroove/nanopore topography regulating stem cell differentiation. Hierarchal microgroove/nanopore topography could promote the polymerization of actin cytoskeleton and activation of ERK and P38 MAPK signaling pathway, which were essential for TAZ activation. Enhanced transcriptional activity and nuclear localization of TAZ could accelerate osteogenesis and impair adipogenesis. 
above results demonstrated that the ERK and p38 MAPK signaling pathway played a key role in hierarchal micro/ nanoscale topography-induced TAZ activation.

Overall, our results indicated that hierarchical microgroove/nanopore topography could facilitate TAZ activation by increasing the nuclear translocation and transcriptional activity of TAZ, which plays a key role in stem cell fate decisions. Mechanistically, TAZ activation triggered by hierarchal microgroove/nanopore topography required both polymerization of the actin cytoskeleton and activation of the ERK and p38 MAPK signaling pathways (Figure 7). Currently, topographical cue-elicited stem cell fate decisions have attracted extensive attention due to their strong prospects in clinical applications. We clarified that TAZ plays a dominant role in this process, which suggests the possibility of promoting TAZ activation to enhance osteogenic differentiation while inhibiting adipogenic differentiation of stem cells, thereby achieving the desired osseointegration around intraosseous implants. However, this orchestrated process requires further characterization. On the one hand, since a previous study revealed that F-actin polymerization was critical for activating the MAPK signaling pathway, ${ }^{59}$ combining the results from the current study, it could be assumed that there is a widespread regulatory network between actin cytoskeleton polymerization and the MAPK signaling pathway in topography-induced TAZ activation. On the other hand, the reciprocal effect of TAZ on osteogenesis and adipogenesis is still unclear. The activation of TAZ could facilitate the bonding of RUNX2 to the promoter OSE2 element of its target gene OCN, finally promoting cell osteogenic differentiation. In contrast, the mechanism by which TAZ activation inhibits adipogenesis through suppression of PPAR $\gamma$ transcriptional activity is still unclear. Daisuke Yamashita reported that SUMOylation of PPAR $22 \mathrm{~K} 107$ was essential for the negative regulation of PPAR $\gamma 2$ transcriptional activity. ${ }^{60}$ Sung Soo Chung reported that SUMOylation of PPAR $\gamma$ could inhibit its binding to the PPRE element of the promoter of its target gene ap2, thereby inhibiting adipogenic differentiation. ${ }^{61}$ Therefore, whether SUMOylation of PPAR $\gamma$ plays an important role in TAZ-inhibited adipogenesis should be further investigated. A comprehensive view of these two aspects will further accelerate the mechanistic investigation of hierarchical micro/nanoscale topography directing cell fate and further lay the foundation for the development of intraosseous implants with the capacity to control reproducible cell behavior.

\section{Acknowledgments}

We extend our thanks to Yu Kong, Lei Cao, Dawei Huo, Rui Li, Zhongye Dai and Xianyou Xia for all their kindness and help.

\section{Funding}

We acknowledge support provided by the National Natural Science Foundation of China (Grant No. 81970958) and the Research Fund of Stomatological Hospital of Tianjin Medical University (Grant No. 2020YKYQ04).

\section{Disclosure}

The authors report no conflicts of interest in this work.

\section{References}

1. Cohen, DM. and Chen CS. Mechanical control of stem cell differentiation. StemBook. Cambridge (MA): Harvard Stem Cell Institute. 2008.

2. Steward AJ, Kelly DJ. Mechanical regulation of mesenchymal stem cell differentiation. J Anat. 2015;227(6):717-731. doi:10.1111/joa.12243

3. Saldana L, Crespo L, Bensiamar F, Arruebo M, Vilaboa N. Mechanical forces regulate stem cell response to surface topography. J Biomed Mater Res A. 2014;102(1):128-140.

4. Dalby MJ, McCloy D, Robertson M, Wilkinson CD, Oreffo RO. Osteoprogenitor response to defined topographies with nanoscale depths. Biomaterials. 2006;27(8):1306-1315. doi:10.1016/j. biomaterials.2005.08.028

5. Biggs MJ, Richards RG, Gadegaard N et al. Interactions with nanoscale topography: adhesion quantification and signal transduction in cells of osteogenic and multipotent lineage. J Biomed Mater Res A. 2009;91(1):195-208.

6. Huang Q, Elkhooly TA, Liu X, et al. Effects of hierarchical micro/ nano-topographies on the morphology, proliferation and differentiation of osteoblast-like cells. Colloids Surf $B$ Biointerfaces. 2016;145:37-45. doi:10.1016/j.colsurfb.2016.04.031

7. Zhao C, Wang X, Gao L, Jing L, Zhou Q, Chang J. The role of the micro-pattern and nano-topography of hydroxyapatite bioceramics on stimulating osteogenic differentiation of mesenchymal stem cells. Acta Biomater. 2018;73:509-521. doi:10.1016/j.actbio.2018.04.030

8. Xu JY, Chen XS, Zhang CY, Liu Y, Wang J, Deng FL. Improved bioactivity of selective laser melting titanium: surface modification with micro-/nano-textured hierarchical topography and bone regeneration performance evaluation. Mater Sci Eng C Mater Biol Appl. 2016;68:229-240. doi:10.1016/j.msec.2016.05.096

9. Li G, Zhou T, Lin S, Shi S, Lin Y. Nanomaterials for Craniofacial and Dental Tissue Engineering. J Dent Res. 2017;96(7):725-732.

10. Zheng G, Guan B, Hu P, et al. Topographical cues of direct metal laser sintering titanium surfaces facilitate osteogenic differentiation of bone marrow mesenchymal stem cells through epigenetic regulation. Cell Prolif. 2018;51(4):e12460. doi:10.1111/cpr.12460

11. Zheng H, Tian Y, Gao Q, et al. Hierarchical micro-nano topography promotes cell adhesion and osteogenic differentiation via integrin alpha2-PI3K-AKT signaling axis. Front Bioeng Biotechnol. 2020;8:463. doi:10.3389/fbioe. 2020.00463

12. Dupont S, Morsut L, Aragona M, et al. Role of YAP/TAZ in mechanotransduction. Nature. 2011;474(7350):179-183. doi:10.1038/ nature 10137

13. Shreberk-Shaked M, Oren M. New insights into YAP/TAZ nucleocytoplasmic shuttling: new cancer therapeutic opportunities? Mol Oncol. 2019;13(6):1335-1341. doi:10.1002/1878-0261.12498 
14. Codelia VA and Irvine KD. Hippo signaling goes long range. Cell. 2012;150(4):669-670. doi:10.1016/j.cell.2012.07.020

15. Lai D and Yang X. BMP4 is a novel transcriptional target and mediator of mammary cell migration downstream of the Hippo pathway component TAZ. Cell Signal. 2013;25(8):1720-1728. doi:10.1016/j.cellsig.2013.05.002

16. Chan SW, Lim CJ, Chen L, et al. The Hippo pathway in biological control and cancer development. J Cell Physiol. 2011;226(4):928-39. doi: $10.1002 /$ jcp. 22435

17. Hong JH, Hwang ES, McManus MT, et al. TAZ, a transcriptional modulator of mesenchymal stem cell differentiation. Science. 2005;309(5737):1074-1078. doi:10.1126/science.1110955

18. Cui CB, Cooper LF, Yang X, Karsenty G, Aukhil I. Transcriptional coactivation of bone-specific transcription factor Cbfa1 by TAZ. Mol Cell Biol. 2003;23(3):1004-1013. doi:10.1128/MCB.23.3.10041013.2003

19. Hwang JH, Lee DH, Byun MR, et al. Nanotopological plate stimulates osteogenic differentiation through TAZ activation. Sci Rep. 2017;7(1):3632. doi:10.1038/s41598-017-03815-5

20. Pan H, Xie Y, Zhang Z, et al. YAP-mediated mechanotransduction regulates osteogenic and adipogenic differentiation of BMSCs on hierarchical structure. Colloids Surf B Biointerfaces. 2017;152:344353. doi:10.1016/j.colsurfb.2017.01.039

21. Zhang Y, Liu N, Shi H, et al. Enhanced osteogenic differentiation of MC3T3-E1 cells on grid-topographic surface and evidence for involvement of YAP mediator. J Biomed Mater Res A. 2016;104(5):11431152.

22. Liu X, Hou W, He L, et al. AMOT130/YAP pathway in topographyinduced BMSC osteoblastic differentiation. Colloids Surf $B$ Biointerfaces. 2019;182:110332.

23. Pereira AM, Tudor C, Pouille P, et al. Plasticity of the MAPK signaling network in response to mechanical stress. PLoS One. 2014;9(7):e101963.

24. Yee KL, Weaver VM and Hammer DA. Integrin-mediated signalling through the MAP-kinase pathway. IET Syst Biol. 2008;2(1):8-15.

25. Yang X, Cai X, Wang J, et al. Mechanical stretch inhibits adipogenesis and stimulates osteogenesis of adipose stem cells. Cell Prolif. 2012;45(2):158-66.

26. Hwang JH, Byun MR, Kim AR, et al. Extracellular matrix stiffness regulates osteogenic differentiation through MAPK activation. PLoS One. 2015;10(8):e0135519. doi:10.1371/journal.pone.0135519

27. Zhang P, Wu Y, Jiang Z, Jiang L, Fang B. Osteogenic response of mesenchymal stem cells to continuous mechanical strain is dependent on ERK1/2-Runx2 signaling. Int J Mol Me. 2012;29(6):1083-1089.

28. Wang L, Li JY, Zhang XZ, et al. Involvement of p38MAPK/NFkappaB signaling pathways in osteoblasts differentiation in response to mechanical stretch. Ann Biomed Eng. 2012;40(9):1884-1894. doi:10.1007/s10439-012-0548-x

29. Codelia VA, Sun G, and Irvine KD. Regulation of YAP by mechanical strain through Jnk and Hippo signaling. Curr Biol. 2014;24 (17):2012-2017.

30. Seo BM, Miura M, Gronthos S, et al. Investigation of multipotent postnatal stem cells from human periodontal ligament. Lancet. 2004;364(9429):149-155.

31. Lin NH, Gronthos S, Bartold PM. Stem cells and periodontal regeneration. Aust Dent J. 2008;53(2):108-121. doi:10.1111/j.18347819.2008.00019.x

32. Griffin MF, Butler PE, Seifalian AM, Kalaskar DM. Control of stem cell fate by engineering their micro and nanoenvironment. World $J$ Stem Cells. 2015;7(1):37-50. doi:10.4252/wjsc.v7.i1.37

33. Dalby MJ, Gadegaard N, Oreffo RO. Harnessing nanotopography and integrin-matrix interactions to influence stem cell fate. Nat Mater. 2014;13(6):558-569. doi:10.1038/nmat3980

34. Sun Z, Guo SS, Fassler R. Integrin-mediated mechanotransduction. $J$ Cell Biol. 2016;215(4):445-456. doi:10.1083/jcb.201609037
35. Burridge K, Turner CE, Romer LH. Tyrosine phosphorylation of paxillin and pp125FAK accompanies cell adhesion to extracellular matrix: a role in cytoskeletal assembly. J Cell Biol. 1992;119(4):893903. doi:10.1083/jcb.119.4.893

36. Chen Q, Kinch MS, Lin TH, Burridge K, Juliano RL. Integrinmediated cell adhesion activates mitogen-activated protein kinases. J Biol Chem. 1994;269(43):26602-26605. doi:10.1016/S0021-9258 (18)47058-5

37. Hall A. Small GTP-binding proteins and the regulation of the actin cytoskeleton. Annu Rev Cell Biol. 1994;10:31-54. doi:10.1146/ annurev.cb.10.110194.000335

38. McBeath R, Pirone DM, Nelson CM, Bhadriraju K, Chen CS. Cell shape, cytoskeletal tension, and RhoA regulate stem cell lineage commitment. Dev Cell. 2004;6(4):483-495. doi:10.1016/S15345807(04)00075-9

39. Sen B, Guilluy C, Xie Z, et al. Mechanically induced focal adhesion assembly amplifies anti-adipogenic pathways in mesenchymal stem cells. Stem Cells. 2011;29(11):1829-1836. doi:10.1002/stem.732

40. Tojkander S, Gateva G, Lappalainen P. Actin stress fibers-assembly, dynamics and biological roles. J Cell Sci. 2012;125(Pt 8):1855-1864. doi: $10.1242 /$ jcs. 098087

41. Geiger B, Spatz JP, Bershadsky AD. Environmental sensing through focal adhesions. Nat Rev Mol Cell Biol. 2009;10(1):21-33. doi: $10.1038 / \mathrm{nrm} 2593$

42. Mendonca G, Mendonca DB, Aragao FJ, Cooper LF. Advancing dental implant surface technology-from micron- to nanotopography. Biomaterials. 2008;29(28):3822-3835. doi:10.1016/j.biomate rials.2008.05.012

43. Zheng H, Tian Y, Gao Q, et al. Hierarchical micro-nano topography promotes cell adhesion and osteogenic differentiation via integrin alpha2-PI3K-AKT signaling axis. Front Bioeng Biotechnol. 2020;8:463. doi:10.3389/fbioe.2020.00463

44. Hong W, Guan KL, The YAP. TAZ transcription co-activators: key downstream effectors of the mammalian Hippo pathway. Semin Cell Dev Biol. 2012;23(7):785-793. doi:10.1016/j.semcdb.20 12.05 .004

45. Liu C, Huang W, Lei Q. Regulation and function of the TAZ transcription co-activator. Int J Biochem Mol Biol. 2011;2(3):247-256.

46. $\mathrm{Gu} \mathrm{K}, \mathrm{Fu} \mathrm{X}$, Tian $\mathrm{H}$, et al. TAZ promotes the proliferation and osteogenic differentiation of human periodontal ligament stem cells via the p-SMAD3. $J$ Cell Biochem. 2019;121(2):1101-1113. doi: $10.1002 /$ jcb. 29346

47. El Ouarrat D, Isaac R, Lee YS, et al. TAZ is a negative regulator of PPARgamma activity in adipocytes and TAZ deletion improves insulin sensitivity and glucose tolerance. Cell Metab. 2020;31(1):162173e165. doi:10.1016/j.cmet.2019.10.003

48. Halder G, Dupont S, Piccolo S. Transduction of mechanical and cytoskeletal cues by YAP and TAZ. Nat Rev Mol Cell Biol. 2012;13(9):591-600. doi:10.1038/nrm3416

49. Elosegui-Artola A, Andreu I, Beedle AEM, et al. Force triggers YAP nuclear entry by regulating transport across nuclear pores. Cell. 2017;171(6):1397-1410e1314. doi:10.1016/j.cell.2017.10.008

50. Sansores-Garcia L, Bossuyt W, Wada K, et al. Modulating F-actin organization induces organ growth by affecting the Hippo pathway. EMBO J. 2011;30(12):2325-2335. doi:10.1038/emboj.2011.157

51. Wada K, Itoga K, Okano T, Yonemura S, Sasaki H. Hippo pathway regulation by cell morphology and stress fibers. Development. 2011;138(18):3907-3914. doi:10.1242/dev.070987

52. Galli C, Piemontese M, Lumetti S, Ravanetti F, Macaluso GM, Passeri G. Actin cytoskeleton controls activation of Wnt/beta-catenin signaling in mesenchymal cells on implant surfaces with different topographies. Acta Biomater. 2012;8(8):2963-2968. doi:10.1016/j. actbio.2012.04.043

53. Cooper JA. Effects of cytochalasin and phalloidin on actin. $J$ Cell Biol. 1987;105(4):1473-1478. doi:10.1083/jcb.105.4.1473 
54. DuFort CC, Paszek MJ, Weaver VM. Balancing forces: architectural control of mechanotransduction. Nat Rev Mol Cell Biol. 2011;12 (5):308-319. doi:10.1038/nrm3112

55. Uhler C, Shivashankar GV. Regulation of genome organization and gene expression by nuclear mechanotransduction. Nat Rev Mol Cell Biol. 2017;18(12):717-727. doi:10.1038/nrm.2017.101

56. Kyriakis JM, Avruch J. Mammalian MAPK signal transduction pathways activated by stress and inflammation: a 10-year update. Physiol Rev. 2012;92(2):689-737. doi:10.1152/physrev.00028.2011

57. Hench LL, Polak JM. Third-generation biomedical materials. Science. 2002;295(5557):1014-1017. doi:10.1126/science.1067404

58. Dupont S. Role of YAP/TAZ in cell-matrix adhesion-mediated signalling and mechanotransduction. Exp Cell Res. 2016;343(1):42-53. doi:10.1016/j.yexcr.2015.10.034
59. Liu Z, Wu H, Jiang K, et al. MAPK-Mediated YAP activation controls mechanical-tension-induced pulmonary alveolar regeneration. Cell Rep. 2016;16(7):1810-1819. doi:10.1016/j.celrep.2016.07.020

60. YAMASHITA D, YAMAGUCHI T, SHIMIZU M, et al. The transactivating function of peroxisome proliferator-activated receptor $\gamma$ is negatively regulated by SUMO conjugation in the amino-terminal domain. Genes to Cells. 2004;9(11):1017-1029. doi:10.1111/j.13652443.2004.00786.x

61. CHUNG SS, AHN BY, KIM M, et al. SUMO modification selectively regulates transcriptional activity of peroxisome-proliferatoractivated receptor $\gamma$ in $\mathrm{C} 2 \mathrm{C} 12$ myotubes. Biochemical Journal. 2011;433(1):155-161. doi:10.1042/BJ20100749

\section{Publish your work in this journal}

The International Journal of Nanomedicine is an international, peerreviewed journal focusing on the application of nanotechnology in diagnostics, therapeutics, and drug delivery systems throughout the biomedical field. This journal is indexed on PubMed Central, MedLine, CAS, SciSearch ${ }^{\mathbb{R}}$, Current Contents ${ }^{\mathbb{R}} /$ Clinical Medicine, $^{2}$
Journal Citation Reports/Science Edition, EMBase, Scopus and the Elsevier Bibliographic databases. The manuscript management system is completely online and includes a very quick and fair peer-review system, which is all easy to use. Visit http://www.dovepress.com/ testimonials.php to read real quotes from published authors. 